\title{
Fungal Endocarditis: A case of Candida glabrata Endocarditis
}

\author{
Faye Sajjadi $^{1}$, Matin Soeizi ${ }^{*}$, Mary Abed ${ }^{1}$ and Tyrone Krause ${ }^{1}$ \\ ${ }^{1}$ Jersey City Medical Center, Jersey City, NJ 07302, USA \\ ${ }^{2}$ St. George's University, USA
}

\section{Introduction}

Infective endocarditis is a rare but life-threatening disease with increasing prevalence. A systemic review by Abdulhak, et al. [1] has shown a crude incidence range from 1.5 to 11.6 cases per 100,000 in high income countries1. Fungal Endocarditis (FE) accounts for a small portion of all cases of endocarditis, only about 1.3-6\% [2]. It has a high mortality and morbidity, ranging within $30-80 \%$. Intracardiac devices or prosthetic valves are important risk factors for fungal endocarditis. The most commonly reported Non-Albican Candida (NAC) causes of Fungal Endocarditis in 2001 was Candida parapsilosis [3]. However that has changed and currently Candida glabrata is the most prevalent NAC pathogenic fungi in humans [4]. In this report we discuss a case of recurrent FE caused by C. glabrata as well as current challenges in treatment. Our patients developed C. glabrata FE once on a native valve and again two years later on his prosthetic valve.

\section{About C. glabrata}

C. glabrata is a unique member of genus Candida species as it is not polymorphic and contains a haploid genome [4]. In the past C. glabrata was considered as a non-pathogenic saprophyte of normal oral flora however that has changed over the past decades. According to Hajjeh and college [5] this change can be contributed to widespread use of immunosuppressive therapy, broad-spectrum antibiotics and antifungals. Currently there is a challenge in treating infectious diseases caused by C. glabrata due to developed resistance.

\section{Case Presentation}

We present a case of a 75 year old male with an multiple comorbidities including hypertension, atrial fibrillation, mitral valve replacement, diabetes, COPD, colon cancer, prostate cancer, and fungemia; however no history of IV drug use or recent immunosuppression. Patient came to the hospital in complaining of shortness of breath on minor exertion. At that time he was on his 42nd day of Micafungin $150 \mathrm{mg}$, which was started at another facility for fungemia. Transthoracic Echocardiogram (TTE) revealed severe pulmonary ypertension, preserved LV function, a $1.0 \times 0.9 \mathrm{~cm}$ mass on the posterior leaflet of the mitral valve, and a $1.5 \times 1.1 \mathrm{~cm}$ mass adherent to the mitral annulus beneath the posterior leaflet of the mitral valve, which was also confirmed on a TEE. During this admission, he was diagnosed with fungemia and fungal Infective Endocarditis (IE) of his native mitral valve. The patient was subsequently taken to the operating room for mitral valve mass removal and valve replacement. Cultures taken intraoperatively from the vegetations and the mitral valve returned growing Candida glabrata, and his treatment was changed to Caspofungin $150 \mathrm{mg}$ IV daily. Patient was discharged on

Caspofungin for a total of 20 days, Micafungin 150 IV daily for 10 days, and prophylactic Fluconazole. He followed up outpatient in one month later, at which time he was taking Fluconazole, and had successfully completed courses for both Caspofungin and Micafungin (Figures 1 and 2).

On November of 2018, the patient presented to the emergency room at another facility with altered mentation and a syncopal episode on his way to church. Upon presenting to that facility he was started on Micafungin $150 \mathrm{mg}$ and the Fluconazole which he had been taking since 2017, was discontinued. He was transferred to our facility after a TTE had revealed recurrence of the vegetation with a large $1.8 \times 1.0 \mathrm{~cm}$ mobile mass on the posterior aspect of his bio-prosthetic valve. He was also found to have acute stroke with multiple small emboli on Magnetic Resonance Imaging (MRI) without significant motor deficits. Patient was again taken to the operating room for his second mitral valve replacement and mass removal. Intra-operative cultures again revealed Candida glabrata. It was concluded that the patient had recurrent $C$. glabrata, this time on a prosthetic mitral valve, despite appropriate treatment and therapy post valve repair in 2017. He was discharged on Caspofungin for a total of 8 weeks and Flucytosine 500mg four times a day for prophylactic treatment (Figures 3 and 4 ).

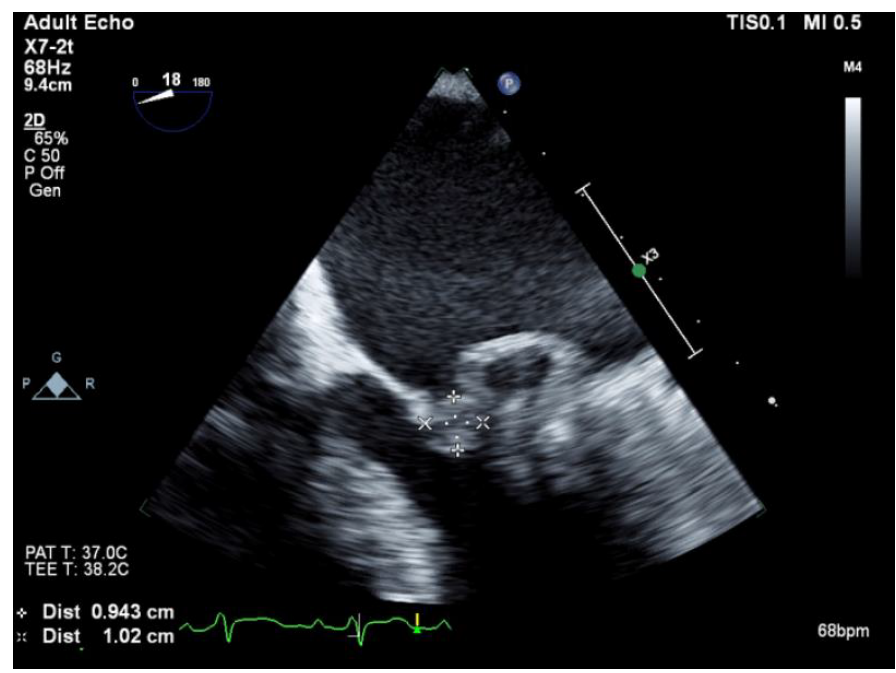

Figure 1. Transesophageal echocardiogram, large $0.9 \times 1.0 \mathrm{~cm}$ vegetation see on the posterior leaflet of the mitral valve

${ }^{\star}$ Correspondence to: Matin Soeizi, Jersey City Medical Center, Jersey City, NJ 07302, USA, E-mail: matinsoeizi@gmail.com

Received: March 26, 2020; Accepted: April 09, 2020; Published: April 13, 2020 


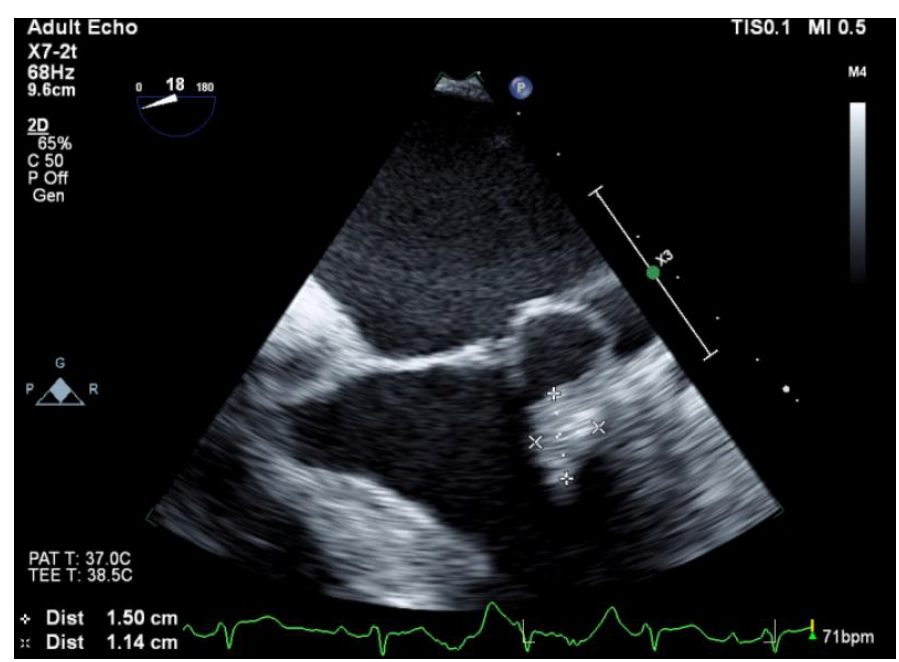

Figure 2. Transesophageal echocardiogram, large $1.5 \times 1.1 \mathrm{~cm}$ mass adherent to the mitral annulus beneath the posterior leaflet of the mitral valve

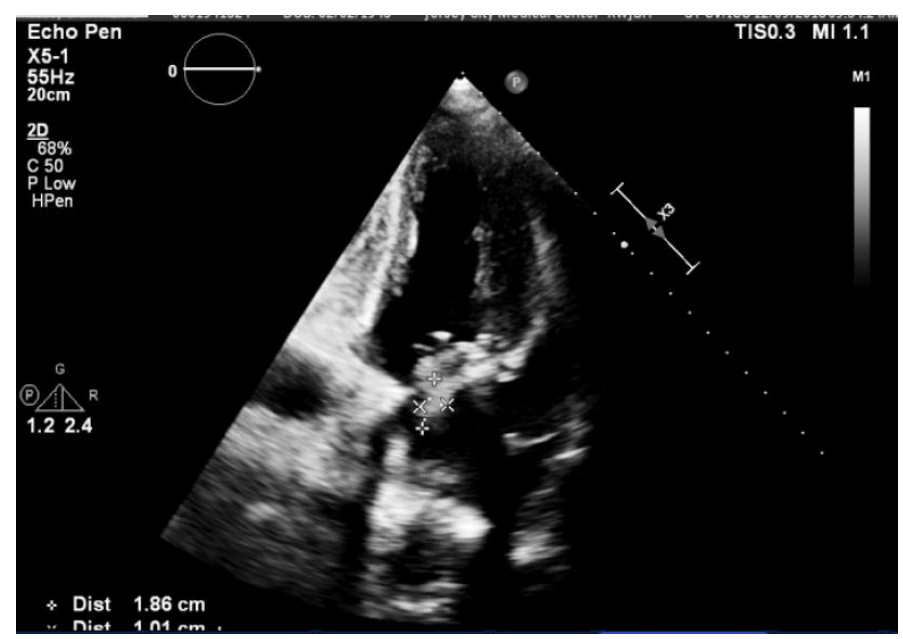

Figure 3. Transthoracic echocardiogram, large $1.8 \times 1.0 \mathrm{~cm}$ mobile mass on the posterior aspect of the bioprosthetic mitral valve

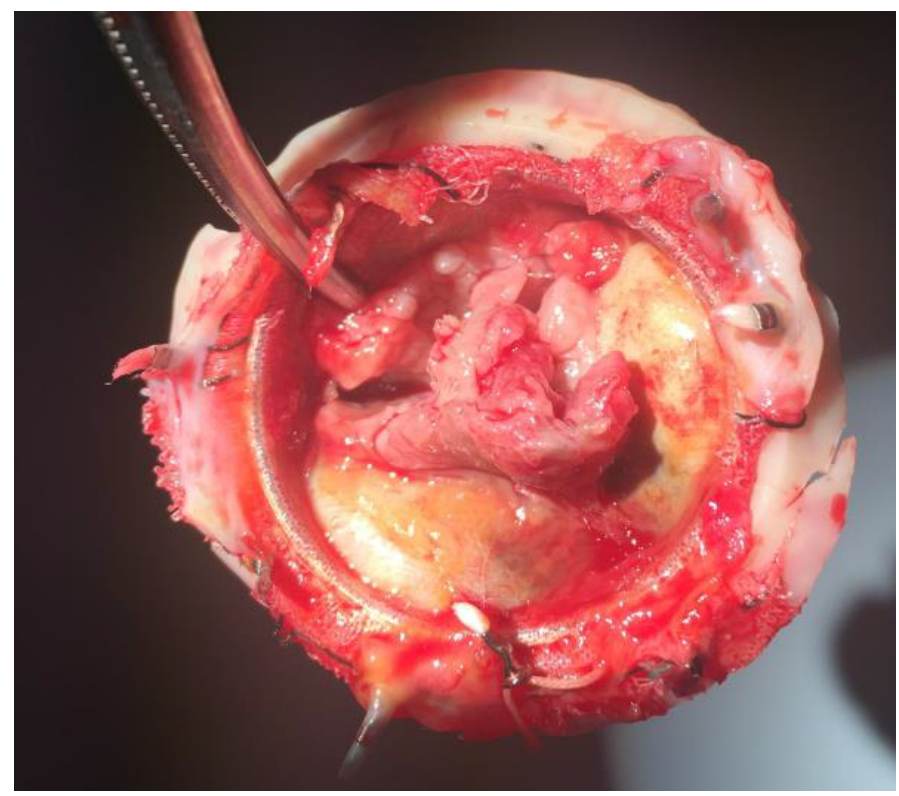

Figure 4. Fungal endocarditis the bio-prosthetic valve

\section{Discussion}

Following the increase in prevalence of IE with C. glabrata further investigation is warranted to determine vulnerable populations, proper treatment regimen, and reduce the rate of recurrence in these patients.

Breaches in natural barriers, catheters, surgery, or prenatal nutrition seems to be the common routes that C. glabrata reaches blood-stream [6]. Fungemia caused by C. glabrata has a significant importance since it has became the second most common fungal infection of the blood stream [7]. Once entered the host C. glabrata attaches to tissues and develops a multilayered biofilm structure. Antifungal agents cannot penetrate the biofilm sufficiently and renders treatment ineffective [4]. Moreover, C. glabrata has the capacity to survive and replicate within the phagosome of phagocytes [8], another factor that contributes to treatment resistance.

C. glabrata is reported to be resistant to all azoles with about $20 \%$ of strains developing resistance to fluconazole during therapy [9]. An extensive study by Spettel K, et al. [10] used Next Generation Sequencing (NGS) and focused on detecting gene mutations in Candida species that might lead to anti-fungal resistance. C. glabrata isolates showed no underlying mutations for echinocandin and azole resistance, as a result the authors suggest that there are still other cellular mechanisms which might contribute to resistance [10].

Including amphotericin B in the treatment regimen of C. glabrata may be beneficial. Rodrigues $\mathrm{R}$, et al. [11] looked into the effect of amphotericin B on pre-formed biofilms and found that C. glabrata had higher susceptibility and biofilm reduction in the presence of amphotericin B compared to fluconazole [11]. However we still need further anti-fungal susceptibility testing and the detection of mutations in resistance patterns. Determining the underlying mechanism of resistance can be helpful in finding the effective treatment against this organism.

\section{Conclusion}

Fungal infective Endocarditis (FE) is a rare condition seen in 1.3$6 \%$ of patients with infective endocarditis [12-15], with Candida and Aspergillus being the two most common etiologies [16]. According to a 16 year study done at Cleveland Clinic, Fungal prosthetic valve endocarditis compromised about $0.1 \%$ of all 10,780 heart valve replacement surgeries [13]. It is a severe opportunistic infection that has a $50 \%$ mortality rate despite appropriate treatment. Diagnosis and treatment of $\mathrm{FE}$ is known to be challenging, partially due to the similarities of clinical presentation to bacterial endocarditis. To add to the complexity of presentation of Candida IE we should mention a reported case of a 57 year old female who presented with myocardial infarction (MI) due to septic embolism to posterolateral branch of the right coronary artery. She was then diagnosed with FIE, however had no signs or symptoms of FIE prior to her MI [20]. Molecular methods used for excised valves are shown to be the most sensitive tool to identify the pathogen causing IE, 3 folds more sensitive than Gram stains and cultures combined [17].

A high recurrence rate is associated with Candida prosthetic endocarditis (CPE). In addition to the rarity of this condition, only about 9 similar cases of FE with multiple surgical repairs have been reported, of which 4 were attributed to medication noncompliance [12$15,18,19]$.

Our patient developed Fungal endocarditis on his native valve followed by late prosthetic valve endocarditis despite proper treatment 
with suppressive anti-fungal therapy. Although there are no definitive guidelines for the treatment of fungal Endocarditis, the combination of both surgical and medical anti-fungal therapy are viewed as the current standard of care. In conclusion, we believe that surgical resection with valve replacement and lifelong anti-fungal medication with addition of amphotericin B are currently the most effective options for treating fungal endocarditis [20].

\section{References}

1. Abdulhak Bin, Baddour ML, Erwin PJ, Hoen B, Chu VH, et al. (2014) Global and regional burden of infective endocarditis, 1990-2010 A systemic review of literature. Global Heart 9: 131-143.

2. Garzoni C, Nobre VA, Garbino J (2007) Candia parapsilosis endocarditis: a comparative review of the literature. Europe Journal of Clinical Microbiology-Infectious Disease 26: 915-926.

3. Ellis ME, Al-Abdely H, Sandridge A, Greer W, Ventura W (2001) Fungal Endocarditis: evidence in the world literature 1965-1995. Clinical Infectious Disease 32: 50-62.

4. Celia F Rodigues, Maria Elisa Rodrigues, Silva Sonia, Henriques Mariana (2017) Candida glabrata Biofilms: How far have we come? J fungi 3: 11.

5. Hajjeh R, Sofair A, Harrison L, Lyon G, Arthington-Skaggs B, et al. (2004) Incidence of bloodstream infections due to candida species and in vitro sesceptilities of isolates collected from 1998 to 2000 in a population-based active surveillance progtam. J Clin Microbiol 42: 1519-1527.

6. Perlroth J, Choi B, Spellberg B (2007) Nosocomial fungal infections: epidemiology, diagnosis, and treatment. Med Mycol 45: 321-346.

7. Tortorano AM, Prigitano A, Lazzarini C, Passera M, Deiana ML, et al. (2013) A 1-year prospective survey of candidemia in Italy and changing epidemiology over one decade. Infection 41: 655-662.

8. Seider K, Brunke S, Schild L, Jablonowski N, Wilson D, et al. (2011) The facultative intracellular pathogen Candida glabrata subverts macrophage cytokine production and phagolysosome maturation. J Immunol 187: 3072-3086.
9. Claudia Colomba, Marcello Trizzino, Claudia Imburgia, Simona Madonia, Lucia Siracusa, et al. (2014) Candida glabrata meningitis and endocarditis: a late severe complication of candidemia. Int J Infect Dis 29. 174-175.

10. Spettel K, Barousch W, Makristathis A, Zeller I, Nehr M, et al. (2019) Analysis of antifungal resistance genes in Candida albicans and Candida glabrata using next generation sequencing. PLoS One 14: e0210397. [Crossref]

11. Célia F Rodrigues, Diana F Alves, Mariana Henriques (2018) Combination of Posaconazole and Amphotericin B in the Treatment of Candida glabrata Biofilms. Microorganisms 6: 123 .

12. Dhakal, Bishnu P (2015) Recurrent candida prosthetic endocarditis over fifteen years managed with medical therapy and four valvular surgeries: a case report and review of literature. Journal of cardiothoracic surgery 10: 105.

13. Muehrcke DD, Lytle BW, Cosgrove DM (1995) Surgical and long-term antifungal therapy for fungal prosthetic valve endocarditis. Ann Thorac Surg 60:538-543.

14. Dismukes WE, Karchmer AW, Buckley MJ, Austen WG, Swartz MN (1973) Prosthetic valve endocarditis. Analysis of 38 cases. Circulation 48: 365-377. [Crossref]

15. Prabhu RM, Orenstein R (2004) Failure of caspofungin to treat brain abscesses secondary to Candida albicans prosthetic valve endocarditis. Clin Infect Dis 39: 12531254.

16. Shi-Min, Yuan (2016) Fungal Endocarditis. Brazilian Journal of Cardiovascular Surgery 31: 252-255.

17. Rice PA, Madico GE (2005) Polymerase chain reaction to diagnose infective endocarditis: will it replace blood cultures? Circulation 111: 1352-1354.

18. Johnston PG, Lee J, Domunski M, Dressler F, Tucker E, et al. (1991) Late recurrent candida endocarditis. Chest 99: 1551-1553.

19. Dhakal, Bishnu P, Tribble, Curtis G, Bergin, et al. (2015) Recurrent Candida prosthetic endocarditis over fifteen years managed with medical therapy and four valvular surgeries: a case report and review of literature. Journal of Cardiothoracic Surgery 10: 105 .

20. Anirudh, Aaron., Upsana, Manchanda-Aaron., Amado X, Freire (2017). Candida Endocarditis presenting as acute myocardial infarction. Ajrccm 196: e4-e6.

Copyright: (C2020 Sajjadi F. This is an open-access article distributed under the terms of the Creative Commons Attribution License, which permits unrestricted use, distribution, and reproduction in any medium, provided the original author and source are credited. 